\title{
Mitochondrial Complex I Encephalomyopathy and Cerebral 5-Methyltetrahydrofolate Deficiency
}

\author{
Author \\ V. T. Ramaekers ${ }^{1}$, J. Weis ${ }^{2}$, J. M. Sequeira ${ }^{3}$ E. V. Quadros ${ }^{3}$, N. Blau ${ }^{4}$ \\ Affiliation \\ 'Division of Pediatric Neurology, University Hospital, RWTH Aachen, Germany \\ Institute of Neuropathology, University Hospital, RWTH Aachen, Germany \\ Department of Medicine and Cell Biology, SUNY-Downstate Medical Center, Brooklyn, New York, USA \\ Division of Clinical Chemistry and Biochemistry, University Children's Hospital, Zürich, Switzerland
}

\author{
Key words \\ - mitochondrial disorder \\ - folate \\ - methyltetrahydrofolate \\ - treatment
}

received 03.06.2007

accepted $\quad 05.09 .2007$

\section{Bibliography}

DOI 10.1055/s-2007-991150

Neuropediatrics 2007;

38: 1-4

(c) Georg Thieme Verlag KG

Stuttgart · New York

ISSN 0174-304X

Correspondence

Dr. V. T. Ramaekers

Division of Pediatric Neurology

University Hospital Aachen

Pauwelsstrasse 30

52074 Aachen

Germany

Tel.: $\mathbf{\square \square}$

Fax: $\mathbf{0}$ 口

vramaekers@skynet.be

\section{Abstract \\ $\nabla$}

Folate transport to the brain depends on ATPdriven folate receptor-mediated transport across choroid plexus epithelial cells. Failure of ATP production in Kearns-Sayre syndrome syndrome provides one explanation for the finding of low spinal fluid (CSF) 5-methyltetrahydrofolate (5MTHF) levels in this condition. Therefore, we suspect the presence of reduced folate transport across the blood-spinal fluid barrier in other mitochondrial encephalopathies. In the present patient with mitochondrial complex I encephalomyopathy a low 5-methyltetrahydrofolate level was found in the CSF. Serum folate receptor autoantibodies were negative and could not explain the low spinal fluid folate levels. The

\section{Introduction}

$\nabla$

Reports of Kearns-Sayre syndrome documented normal plasma folate levels associated with isolated lowering of CSF 5-methyltetrahydrofolate (5MTHF), which was believed to result from failure of adequate ATP production necessary for the active ATP-dependent folate transport across the blood-brain barrier $[2,5,7,12]$. In two deceased patients with Kearns-Sayre syndrome autopsy studies documented oncocytic transformation of choroid plexus epithelial cells with an abundance of deletions in mitochondrial DNA and decreased expression of mitochondrial DNA encoded proteins [12]. However, one report demonstrated not only low CSF 5MTHF concentrations, but also low plasma folate concentrations [1].

Therefore, we investigated the folate metabolism in a patient with mitochondrial complex I encephalomyopathy, as well as the effect of addon treatment with folinic acid, radical scavengers and cofactors of complex I [11]. epileptic seizures did not respond to primidone monotherapy, but addition of ubiquinone-10 and radical scavengers reduced seizure frequency. Add-on treatment with folinic acid led to partial clinical improvement including full control of epilepsy, followed by marked recovery from demyelination of the brainstem, thalamus, basal ganglia and white matter. Cerebral folate deficiency is not only present in Kearns-Sayre syndrome but may also be secondary to the failure of mitochondrial ATP production in other mitochondrial encephalopathies. Treatment with folinic acid in addition to supplementation with radical scavengers and cofactors of deficient respiratory enzymes can result in partial clinical improvement and reversal of abnormal myelination patterns on neuro-imaging.

\section{Patient and Methods \\ $\nabla$}

After diagnosis of mitochondrial encephalomyopathy in the patient, further investigations were performed to study folate transport mechanisms across the blood-brain barrier. After informed parental consent, CSF specimens were collected between 8:30 and 10:00 A.M. and analyzed for the end and intermediary metabolites of the biogenic monoamines dopamine and serotonin, pterins and 5-methyltetrahydrofolate (5MTHF). Simultaneously we also determined serum folate, vitamin B12 and homocysteine levels. The presence of blocking autoantibodies against the folate receptors was investigated using previously described methods [9].

Following add-on treatment with folinic acid combined with the previously started treatment of the radical scavengers vitamin $\mathrm{C}$ and $\mathrm{E}$ and ubiquinone-10 as co-factor of the deficient respiratory enzyme complex I, the patient was followed up for a period of 3 years. 
Cerebral MRI investigations were performed before and after treatment.

\section{Case Report \\ $\nabla$}

After a normal pregnancy the patient, now a 6-year-old boy, was born by Caesarian section after prolonged labour with an Apgar score of 7-7-9 after 1, 5 and 10 minutes, respectively, and an umbilical arterial pH of 7.25. His birth weight was $3950 \mathrm{~g}$ and his length $54 \mathrm{~cm}$. Both parents were non-consanguineous and healthy, except for severe psoriasis of the mother. Because of feeding difficulties and generalized muscle weakness since birth, the patient was referred for a muscle biopsy after finding marked elevation of plasma lactate ( $8.2 \mathrm{mM}$; normal range 1-1.8).

Morphological analysis of the muscle biopsy revealed myofibrillar anomalies and hypertrophic type I fibres in the presence of normal and atrophic type II fibres. Electron microscopy showed the presence of myofibrillary abnormalities as well as swelling and hypertrophy of mitochondria.

Biochemical investigations of a fresh muscle sample showed marked reduction of oxidation rates after preincubation with various ${ }^{14} \mathrm{C}$-radiolabelled substrates, as well as a reduced production rate of ATP + creatine phosphate from pyruvate. Measurement of the activities of the pyruvate dehydrogenase complex and respiratory chain enzymes revealed an isolated moderate deficiency of complex I NADH-Q10 oxidoreductase at $50 \mathrm{mU} / \mathrm{U}$ CS (normal reference values for complex I activity: $70-250 \mathrm{mU} / \mathrm{U}$ CS [citrate synthetase]). Fibroblast cultures showed normal respiratory enzyme activities. Molecular genetic investigations could rule out deletions and mutations of mitochondrial DNA, tRNA for leucine and lysine, and the common mutations found in MELAS, MERRF and Leigh/NARP. Analysis of nuclear genes encoding for the subunits of the mitochondrial complex I have ruled out mutations of NDUFS 1, 2, 4 and 7. Further investigation of other nuclear encoded complex I units are pending.

From birth, neurodevelopment was characterized by generalized muscle hypotonia and weakness with marked delay in acquisition of motor milestones, ataxia, development of pyramidal deficits in the lower limbs and the onset of generalized and focal epileptic seizures from the age of 1 year. At the age of 1 year, anticonvulsant drug treatment with primidone failed to control seizures. MRI of the brain at the age of 14 months showed fronto-temporal atrophy with bilateral extensive signs of leukoencephalopathy affecting the supratentorial white matter, ventral part of the internal capsule, cerebral peducles and brainstem. $\mathrm{T}_{2}$-weighted sequences showed bilateral hyperintensive signal changes of the thalamus, hypothalamus, quadrigeminal plate and dorsal pons. Minor bilateral signal changes were present at the medial lenticular nucleus. CSF analysis at the age of 1.5 years showed moderate lactate elevation at $3.6 \mathrm{mM}$ (reference $<1.5$ ) and diminished CSF 5MTHF at $38.5 \mathrm{nM}$ (normal reference range 64-182) in the presence of normal values for the intermediary and end metabolites of the monoamines dopamine and serotonin (5-hydroxytryptophane, 5-hydroxyindoleacetic acid, homovanillic acid, L-dopa, 3-O-methyldopa) and pterins. Serum folate, vitamin B 12 and homocysteine were normal. Serum blocking autoantibodies against the folate receptor were absent.

From the age of 6 months a multivitamin preparation without folate was started and had no effect. From the age of 1 year and
2 months this multivitamin preparation was replaced by the complex I cofactor ubiquinone- $10(2-3 \mathrm{mg} / \mathrm{kg} /$ day $)$ in combination with high doses of the radical scavengers vitamin C $(10 \mathrm{mg} / \mathrm{kg})$ and $\mathrm{E}(25 \mathrm{U} / \mathrm{kg})$. The frequency of akinetic and adverse motor seizures diminished but the other clinical signs and symptoms did not respond.

From the age of 1 year and 10 months, folinic acid $(1.2 \mathrm{mg} / \mathrm{kg}$ $\mathrm{BW})$ and riboflavin $(0.5-1 \mathrm{mg} / \mathrm{kg})$ were added. After the addition of folinic acid and riboflavin to the previously mentioned treatment the seizures became fully controlled after 1 year, with normalization of the EEG. Hypotonia and ataxia improved and the patient was able to sit alone after 4 years treatment with folinic acid. However, cognitive deficits and pyramidal signs of the lower limbs persisted.

MRI follow-up at the age of 4 years and 11 months, following treatment with folinic acid, cofactors of complex I and radical scavengers for more than three years, showed marked reversal of the earlier observed signs of de-/hypomyelination and the absence of an elevated lactate signal on MR spectroscopy (० Fig. 1).

\section{Results of treatment \\ $\nabla$}

After add-on treatment with folinic acid there was partial clinical improvement with reduction of seizures, hypotonia and ataxia, whereas cognitive deficits and pyramidal signs in the lower limbs persisted. MRI follow-up showed reversal of abnormal myelination of brainstem, basal ganglia and white matter, while MR spectroscopy showed the absence of an elevated lactate peak.

\section{Discussion \\ $\nabla$}

This case report describes a severe mitochondrial complex I encephalomyopathy manifesting with psychomotor retardation, weakness, hypotonia and ataxia, spastic diplegia and refractory seizures and MRI changes of leukoencephalopathy with demyelination. The clinical picture of the patient with mitochondrial encephalopathy due to complex I deficiency resembles that of the children with the infantile-onset CFD syndrome ([3, 4,6, 8,9,13]; O Table 1). The normal plasma homocysteine and normal hematological investigations with the absence of macrocytic anemia in the patient never suggested a disorder of folate metabolism. However, the 5MTHF measurement in CSF was low. The presence of low CSF folate levels prompted the analysis for blocking autoantibodies to the folate receptor in the serum which was found negative. The reduced transport of

Table 1 Clinical findings in the patient compared to the major clinical features in the infantile-onset CFD syndrome

\begin{tabular}{|lc}
\hline Clinical feature & Patient \\
\hline deceleration of head growth & - \\
\hline agitation, insomnia & + \\
\hline psychomotor retardation & + \\
\hline autistic features & - \\
epilepsy & + \\
\hline pyramidal signs & + \\
\hline extrapyramidal signs & - \\
\hline ataxia and hypotonia & + \\
\hline
\end{tabular}




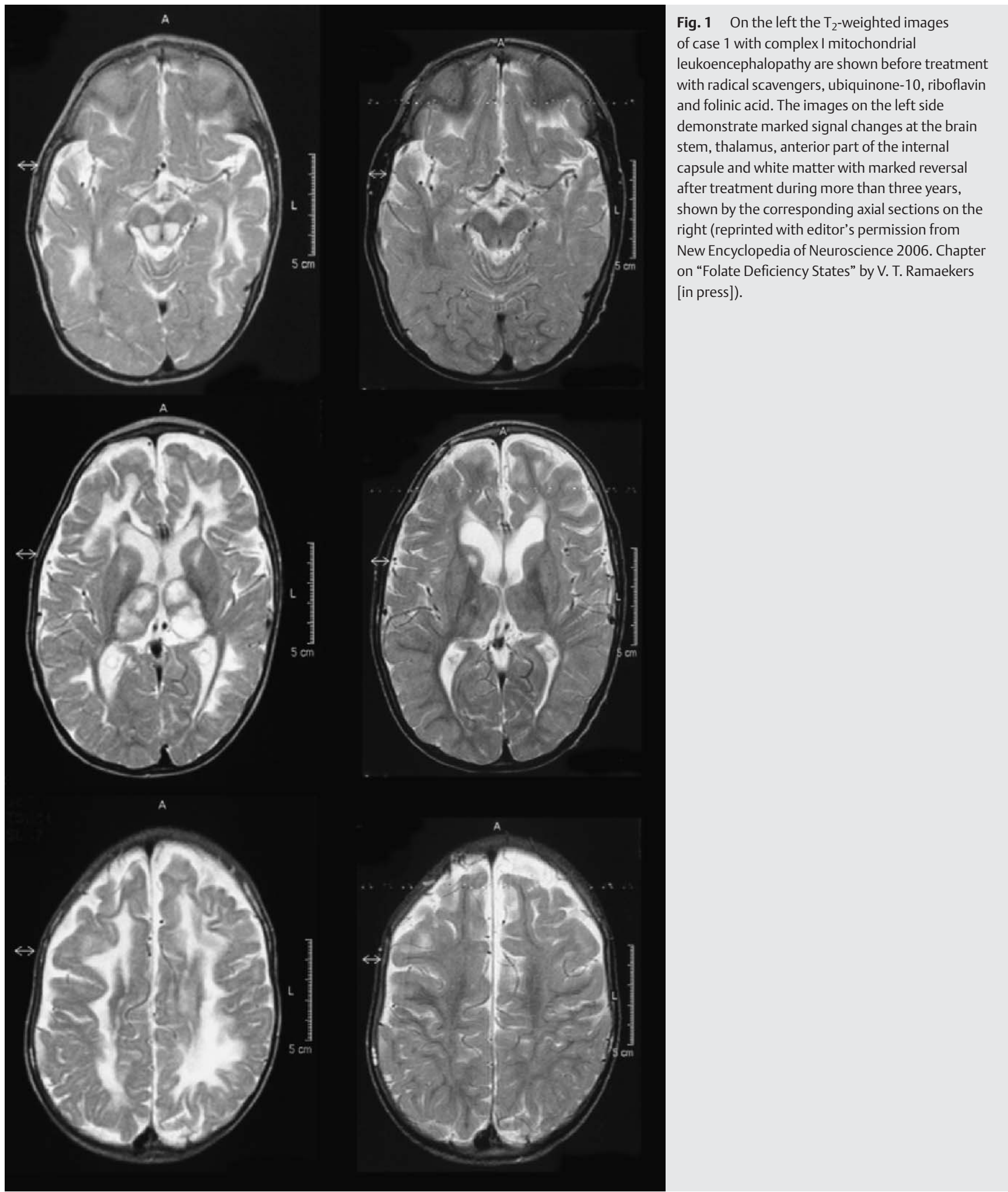

5MTHF across the choroid plexus in this mitochondrial disorder can be tentatively attributed to a failure of ATP production. The therapeutic effect of the combination of folinic acid and radical scavengers could be demonstrated in vivo by the control of refractory epilepsy and by the reversal of leukoencephalopathic changes in the patient who received treatment for 3 years. In addition, the absence of an elevated lactate on follow-up by MR spectroscopy also confirms the beneficial effect of this treatment. Previous reports on treatment with cofactors of deficient respiratory chain complexes combined with antioxidants showed conflicting results with some anecdotal reports of a curative effect [10]. A recent report also documented a dramatic reversal of white matter demyelination in a patient with KearnsSayre syndrome with low CSF folate who received folinic acid over a period of 1 year [7]. Further studies are needed to confirm the effect of early intervention with folinic acid combined with 
antioxidants and respiratory chain cofactors among the heterogeneous group of mitochondrial disorders affecting the central nervous system.

\section{Acknowledgments}

We thank Mrs. L. Kierat for performing the CSF folate studies and Mrs. H. Mader for skillful technical assistance. This work has been supported in part by a R21 grant from the NIH to E. V. Quadros.

\section{References}

1 Allen RJ, DiMauro S, Coulter DL, Papadimitriou A, Rothenberg SP. KearnsSayre syndrome with reduced plasma and cerebrospinal fluid folate. Ann Neurol 1983; 13: 679-682

2 Dougados M, Zittoun J, Laplane D, Castaigne P. Folate metabolism disorder in Kearns-Sayre syndrome. Ann Neurol 1983; 13: 687

3 Fillano JJ, Goldenthal MJ, Rhodes CH, Marin-Garcia J. Mitochondrial dysfunction in patients with hypotonia, epilepsy, autism, and developmental delay: HEADD syndrome. J Child Neurol 2002; 17: 435-439

4 Loeffen JL, Smeitink JA, Trijbels JM, Janssen AJ, Triepels RH, Sengers RC, Heuvel LP van den. Isolated complex I deficiency in children: clinical, biochemical and genetic aspects. Hum Mutat 2000; 15: 123-134
5 Macron JM, Mizon JP, Rosa A. Disorders of folate metabolism in the Kearns-Sayre syndrome. Rev Neurol (Paris) 1983; 139: 673-577

6 Moroni I, Bugiani M, Bizzi A, Castelli G, Lamantea E, Uziel G. Cerebral white matter involvement in children with mitochondrial encephalopathies. Neuropediatrics 2002; 33: 79-85

7 Pineda M, Ormazabal A, López-Gallardo E, Nascimento A, Solano A, Herrero MD, Vilaseca MA, Briones $P$, Ibániez L, Montoya J, Artuch $R$. Cerebral folate deficiency and Leukoencephalopathy caused by mitochondrial DNA deletion. Ann Neurol 2006; 59: 394-398

8 Ramaekers VT, Hausler M, Opladen T, Heimann G, Blau N. Psychomotor retardation, spastic paraplegia, cerebellar ataxia and dyskinesia associated with low 5-methyltetrahydrofolate in cerebrospinal fluid: a novel neurometabolic condition responding to folinic acid substitution. Neuropediatrics 2002; 33: 301-308

9 Ramaekers VT, Rothenberg SP, Sequeira J, Opladen T, Blau N, Quadros $E V$, Selhub J. Autoantibodies to folate receptors in the cerebral folate deficiency syndrome. N Engl J Med 2005; 352: 1985-1991

10 Schapira AH. Mitochondrial disease. Lancet 2006; 368: 70-82

11 Sheu SS, Nauduri D, Anders MW. Targeting antioxidants to mitochondria: a new therapeutic direction. Biochim Biophys Acta 2006; 1762: 256-265

12 Tanji K, Schon EA, DiMauro S, Bonilla E. Kearns-Sayre syndrome: oncocytic transformation of choroid plexus epithelium. J Neurol Sci 2000; 178: 29-36

13 Valanne L, Ketonen L, Majander A, Suomalainen A, Pihko H. Neuroradiologic findings in children with mitochondrial disorders. AJNR Am J Neuroradiol 1998; 19: 369-377 